\title{
INVESTIGACIÓN Y PRODUCCIÓN DE CONOCIMIENTO EN EDUCACIÓN MATEMÁTICA: UNA CUESTIÓN DE MERCADO, PODER Y ESTÉTICA
}

\author{
RESEARCH AND KNOWLEDGE PRODUCTION IN MATHEMATICS EDUCATION: \\ AN ISSUE OF MARKET, POWER AND AESTHETICS \\ PESQUISA E PRODUÇÃO DE CONHECIMENTO EM EDUCAÇÃO MATEMÁTICA: \\ UMA QUESTÃO DE MERCADO, PODER E ESTÉTICA
}

\author{
Alex Montecino \\ Investigador independiente, Santiago, Chile \\ Recibido: 07/06/2021 - Aceptado: 19/07/2021 - Publicado: 01/12/2021 \\ Remita cualquier duda sobre esta obra a: Alex Montecino. \\ Email:alex.montecino.em@gmail.com
}

\begin{abstract}
RESUMEN
Este artículo pretende abrir una discusión sobre las condiciones que hacen posible ciertos entendimientos sobre qué es la investigación y la (re)producción de conocimiento dentro del área. A partir de una problematización de las redes discursivas en la que circulan formas de ser y actuar deseados se desempaca formas de conducir las prácticas investigativas y el devenir del investigador. Investigando la investigación, como estrategia analítica, este artículo busca desplazar el foco de atención más allá de una mirada positivista o neopositivista de la producción del conocimiento, con ello posicionar la mirada en la lógica de mercado y su dinámica, el poder, el autor y la estética. Ergo, se muestra que las normas que delinean la investigación y la producción de conocimiento no tienen autoridad por sí mismas, más bien estas se configuran como parte de redes de poder. Por otra parte, se acentúa el hecho que la investigación debe abrirnos posibilidades de acción y reacción, ya sea estas incomodas o gratas.
\end{abstract}

Palabras clave: Investigación; (Re)producción de conocimiento; Deseo; Poder; Estética.

\begin{abstract}
The article aim is to open a discussion on conditions that make possible understanding about research and (re)production of knowledge within the area. The articulation of discursive networks is problematized, in order to unpack the conduction of investigative practices and the becoming the researcher, as well as desired ways of being and acting. By deploying researching research, as an analytical strategy, this paper seeks to move the focus beyond a positivist or neo-positivist view or understanding of the production of knowledge. It is positioning the gaze on the market logic and its dynamics, power, the author and the aesthetic. Therefore, it is drawn conclusions
\end{abstract}


regarding how norms that delineate research and the production of knowledge do not have authority by themselves, rather they are configured as part of the power networks, moreover, it is emphasizing the fact that research should open us possibilities of action and reaction, whether these are uncomfortable or pleasant.

Keywords: Research; (Re)production of knowledge; Desire; Power; Aesthetic

\section{RESUMO}

O objetivo do artigo é abrir uma discussão sobre as condiçóes que possibilitam o entendimento sobre a pesquisa e (re)produção do conhecimento na área. A articulação das redes discursivas é problematizada, a fim de pensar a condução das práticas investigativas e o tornar-se pesquisador, bem como as formas desejadas de ser e agir. Ao implantar uma pesquisa de pesquisa, como estratégia analítica, este artigo procura mover o foco para além de uma visão positivista ou neo-positivista ou compreensão da produção de conhecimento. É posicionar o olhar sobre a lógica do mercado e sua dinâmica, poder, autor e estética. Portanto, tira-se conclusões a respeito de como as normas que delineiam a pesquisa e a produção do conhecimento não têm autoridade por si mesmas, mas se configuram como parte das redes de poder, aliás, é enfatizar que a pesquisa deve nos abrir possibilidades de ação. e reação, sejam elas desagradáveis ou agradáveis.

Palavras-chave: Pesquisa; (Re)produção de conhecimento; Desejo; Poder; Estética.

\section{PRODUCCIÓN DE CONOCIMIENTO: UNA CUESTIÓN DE ESTUDIO}

Dentro del campo en educación matemática, la producción y reproducción —-(re)producción en adelante- de conocimiento ha devenido en un asunto de estudio e interés en los últimos años. Prueba de ello son las investigaciones que abordan cuestiones de calidad en el área, poniendo la atención en diferentes aspectos que se entrecruzan en los procesos de publicación dentro de las revistas (ver Nivens \& Otten, 2017; Toerner \& Arzarello, 2012; Williams \& Leatham, 2017). A la luz de estas discusiones, se han dado forma a diferentes rankings de revistas de la especialidad, como es el caso del ranking de revistas anglosajonas propuesto por la investigación de Williams y Leatham (2017); y el ranking de revistas iberoamericanas propuesto por la investigación de Andrade-Molina et al. (2020). También es posible encontrar estudios, desde una posición más crítica, que problematizan la configuración de un espacio de segregación y exclusión en el proceso de producción y diseminación del conocimiento.

Dicho espacio de segregación y exclusión toma forma desde: (a) El poder que generan los rankings de revistas y su entrelazamiento con aspectos económicos y políticos de la academia (AndradeMolina et al., 2020); (b) El establecimiento del inglés como lengua franca para la publicación y divulgación del conocimiento, conllevando esto a favorecer un monoculturalismo en la investigación, así como el surgimiento de ciertas normas que nos indican como educadores matemáticos aquello que le da valor a una investigación (Meaney, 2013). Como consecuencia, los investigadores tienden a referenciar investigaciones publicadas en revistas anglosajonas para ser tomados en serio y en cuenta en los diferentes escenarios en los que se mueven (Meaney, 2013); (c) El surgimiento de un "tipo ideal” de artículo de investigación. Si bien este tipo de artículo representa una comprensión muy estrecha y rígida de la investigación, ya que no refleja la realidad multifacética del campo y pone en peligro de desarrollo y 
renovación de la investigación (Niss, 2018), a su vez, tiene el potencial de conducir las formas en la que se entiende y se debe escribir un artículo de investigación; (e) Los sesgos que se configuran en el proceso de revisión por pares, los cuales pueden darse por, el contenido (tema, métodos, orientación teórica/metodológica, resultados) del trabajo, el origen de quien escribe, los alcances e interés de la revista e identidad de los investigadores (ver Lee et al., 2013; Williams \& Leatham, 2017).

A fines de septiembre del 2021 en Mathematics Education and Society Conference, un grupo de investigadores (incluido el autor de este trabajo) desarrolló un simposio titulado Publish or perish: power and bias in peer review processes in mathematics education journals, cuyos objetivos fueron explorar los sesgos en el proceso de publicación, así como, encontrar y explorar formas de abordar dichos sesgos en los procesos de revisión por pares, a partir de discusiones con académicos. Uno de los puntos discutidos fue la normalización de una supuesta superioridad, de ciertos grupos por sobre otros, con respecto a las formas de hacer investigación o sobre lo que se investiga. Esta supuesta superioridad agudiza aún más la configuración de los procesos de producción y diseminación del conocimiento como un espacio de segregación y exclusión.

Estas discusiones y tensiones no han sido exclusivas del área. También se han hecho presentes en el campo de la filosofía de la ciencia. Si consideramos al positivismo y a la hermenéutica, estas si bien no niegan la existencia y la relevancia de la (re)producción de conocimiento, más bien pareciera que la invisibilizan al desplazar su discusión fuera del propio territorio crítico en el que se debería transitar para pensar y repensar concepciones filosóficas que sustenta lo científico y su devenir, como es el caso de la verdad. En esta línea cabe preguntarse: ¿Cómo estamos concibiendo, como educadores matemáticos, aquellas concepciones? ¿Cómo estas concepciones nos llevan a actuar de una u otra forma dentro del área? ¿Cómo hemos llegado a entender y pensar la investigación de la forma que se hace en la actualidad? ¿Qué es y cómo debe ser una investigación?

Si nos posicionamos desde los trabajos de Deleuze, en particular en los que se establecen cuestionamientos sobre la epistemología y la filosofía de las ciencias (por ejemplo, Deleuze \& Guattari, 1977), vemos la necesidad, en primer lugar, de reabrir asuntos que se han dado como saldados, al punto de llegar a ser incuestionables - deviniendo en verdades naturalizadas-, como es el caso de lo que se entiende por ciencia o conocimiento científico, en nuestro caso específico, conocimiento dentro de la educación matemática. Por otra parte, se vuelve necesario repensar la producción científica al poner la atención sobre el entramado donde lo social, económico y político se entreteje —al ser entendidos estos como líneas de distinta naturaleza, de distintos tipos, intensidades, velocidades y direcciones (Deleuze, 1992). El reabrir y repensar estos asuntos conlleva reconocer a la ciencia como no neutral, ya sea por su intento de representar/controlar la naturaleza y de gobernar lo social, o por su intento de dotar de un valor agregado a quien tiene y maneja este conocimiento.

Ahora bien, si miramos lo que pasa dentro de la educación matemática con respecto a la (re)producción de conocimiento, existe una línea de desarrollo que se sustenta en argumentos sobre la 
relevancia e importancia del conocimiento matemático — así como, del conocimiento científico- y su enseñanza y aprendizaje para la sociedad moderna. Dándose por sentado, en estos argumentos, principios de neutralidad y epistémicos que conforman maneras de sustentar la (re)producción de conocimiento. Se argumenta que las matemáticas, junto con la lectura y las ciencias, son dominios que se consideran centrales a la hora de favorecer ciertos conocimientos y desarrollar determinadas habilidades/competencias claves para la formación de un ciudadano moderno. Un ciudadano que debe ser capaz de navegar en una sociedad cada vez más tecnológica, así como en las actividades propias del mundo laboral y en procesos sociales y políticos (OCDE, 2014a). Adicionalmente, la OCDE (2014a) afirma que el fomentar la excelencia en estos tres dominios - matemáticas, lectura y ciencias - es crucial para el desarrollo de un país que quiere estar a la vanguardia de una economía global, competitiva y basada en el conocimiento.

En particular, las matemáticas y las ciencias - ya sea su desarrollo, como su enseñanza y aprendizaje - han sido asociadas, por diferentes esferas sociales, con la capacidad de mejorar al ser humano y a la sociedad en general. Narrativa sustentada en la supuesta "superioridad epistemológica [de las matemáticas y de las ciencias] y su poder como herramienta para competir en una economía del conocimiento globalizado" (Valero, 2017, p. 120), dotando a quienes las aprenden de una forma de ver y entender el mundo mediante un ojo entrenado (Andrade-Molina, 2017) y de conocimientos, de formas de razonar y de aptitudes consideradas valiosas para la sociedad. Un ejemplo concreto de esta narrativa es la idea que circula en diferentes reportes de la OCDE, donde se señala que el desarrollo del conocimiento y las habilidades matemáticas otorga ciertos atributos morales y éticos a la persona. En esta línea, se afirma que:

Las habilidades básicas en matemáticas tienen un gran impacto en las oportunidades de vida de las personas [...] las habilidades matemáticas deficientes limitan severamente el acceso de las personas a trabajos mejor remunerados y más gratificantes [...] Más allá de eso, la encuesta muestra que las personas con habilidades sólidas matemáticas es más probable que las sean voluntarias, se vean a si mismas como actores en lugar de objetos de procesos politicos, y es incluso más probable que confien en otros. La equidad, la integridad y la inclusión en la politica pública dependen de las habilidades de los ciudadanos. (OCDE, 2014b, p. 6)

En otras palabras, una persona con altas habilidades matemáticas - y sin duda con habilidades científicas, como se afirma OCDE (2014b, 2019a, 2019b, 2019c) — es más probable que se convierta en un mejor ciudadano (ver OCDE, 2014b, 2019a, 2019b). En la actualidad se está deseando un sujeto constructivo, comprometido y reflexivo que utilice las matemáticas y las ciencias como una herramienta para resolver problemas de la vida cotidiana (OCDE, 2014c, 2019a, 2019b), y para comprender y controlar su realidad social y física (OCDE, 2010). Narrativas que también se ven presente en gran parte de la 
investigación del área, así como en discursos que hacen alusión al propósito formativo de las matemáticas y de las ciencias en la escuela. Lo que sin duda direcciona y matiza la (re)producción del conocimiento.

En los estudios de cohorte más socio culturales, esta narrativa se ha vuelto explicita, como se puede ver en los trabajos de Gutstein (2006, 2012), en los que se sostiene la necesidad de propiciar condiciones para que los estudiantes, mediante las matemáticas, estén preparados para leer y escribir el mundo, y con ello criticar y desafiar la injusticia, las estructuras y actos opresivos (Gutstein, 2006), es decir, usar las matemáticas como un arma para la lucha y transformación social (Gutstein, 2012). Por otra parte, se afirma que la adquisición de ciertas competencias es clave para el ciudadano y sociedad, al respecto Niss et al. (2007) nos afirman que la competencia de modelación "potencia el conocimiento, a su vez asegura el avance de la sostenibilidad del bienestar de la salud, la educación y el medio ambiente, y la reducción de la pobreza y desventajas” (pp. 17-18), por lo que es necesario fomentarla. El conocimiento matemático se ha establecido indispensable para todo ciudadano, al brindar un lenguaje para la ciencia y la tecnología y una forma válida de pensar y argumentar (Smid, 2014), fundamentales para el desarrollo de la sociedad (OCDE, 2014a), al punto de favorecer a los trabajadores para que se sientan más "empoderados para hacer preguntas inteligentes sobre la práctica laboral con miras a mejorar o evitar errores graves" (FitzSimons, 2019, p. 43).

Desde el contexto chileno, el Ministerio de Educación (MINEDUC) establece que las matemáticas están en el currículo escolar para (a) enriquecer la comprensión de la realidad, (b) favorecer estrategias para la resolución de problemas, y (c) contribuir con el desarrollo del pensamiento crítico y autónomo de los estudiantes (MINEDUC, 2012). Con respecto a las ciencias, en el currículo escolar establece que estas deben servir para: (a) fomentar el proceso de investigación, con ello construir nuevos conocimientos, (b) favorecer estrategias para la resolución de problemas y argumentar, y (c) tributar al desarrollo de herramientas para ayudar a identificar problemas, recopilar y analizar datos, modelar fenómenos y probar las posibles soluciones para la vida profesional y diaria MINEDUC (2021).

La narrativa dominante posiciona al pensamiento y razonamiento matemático y científico en un lugar privilegiado dentro de la sociedad, ya que estos se entienden como un prerrequisito para la participación social, cultural, económica y política. Problematizar los efectos de poder -en terminología Foucaultiana - de esta narrativa se vuelve clave en una agenda investigativa, ya que nos remite a un terreno donde impera una racionalidad que configura un entramado que norma nuestros entendimiento y formas de pensar, estableciendo las "reglas y estándares que vigilan los límites de lo que es posible y no es posible pensar y hacer, lo que se da como sentido común y natural que gobierna la conducta y que simultáneamente excluye y abyecta" (Popkewitz et al., 2017, p. 3). Entramado que, en la actualidad, se permea con los discursos positivistas o neopositivistas en los que navegan los entendimientos contemporáneos sobre lo que se considera o no se considera como conocimiento y ciencia - así como el acto de creación y la divulgación del conocimiento. 


\section{INVESTIGANDO LA INVESTIGACIÓN COMO ESTRATEGIA ANALÍTICA}

Este artículo no tiene la finalidad de hacer una revisión de la investigación en educación matemáticas y con ello ofrecer un panorama general de la investigación. Dentro del área podemos encontrar diferentes contribuciones que tienen aquel propósito, como es el caso, por ejemplo, de los trabajos de Lerman (2001) y Stahnke et al. (2016), quienes hacen una revisión sistemática de la investigación que tiene como foco de estudio al profesor de matemáticas. Más bien, en este artículo se pretende abrir una discusión sobre las condiciones que hacen posibles ciertos entendimientos sobre lo que se considera como investigación y la producción de conocimiento dentro del área. Para ello, es necesario un posicionamiento crítico hacia los juegos de verdad en los que se (re)producen formas de pensar, concebir e investigar dentro de sistemas de conocimiento y poder. Consecuentemente, la investigación y la (re)producción de conocimiento se problematizan mediante la deconstrucción de la red discursiva en la que circulan formas de ser y actuar deseados, normando nuestras prácticas investigativas y nuestro devenir como investigadores.

En otras palabras, lo que se busca en este trabajo es investigar la investigación (Pais \& Valero, 2012) para evidenciar cómo la investigación y el acto de investigar configura condiciones para pensar la producción y reproducción de conocimiento al dar forma nociones sobre los tipos de personas y prácticas deseadas y, al mismo tiempo, nociones sobre los tipos de personas y prácticas temidas. Este doble gesto constituye una técnica importante para gobernar las subjetividades a través del conocimiento, como ha señalado Foucault (1991). En consecuencia, la investigación en educación matemática no es una actividad inocente, ya que esta produce lenguajes y herramientas que dan forma a lo que vemos y decimos dentro del área (Pais \& Valero, 2012), así como, también da forma a lo deseable y, al mismo tiempo, articula las formas de lograrlo (Popkewitz, 2011).

Investigar la investigación es, en este caso, un movimiento político y crítico para problematizar la circulación de discursos, verdades y relaciones de poder dentro de los cuales se constituyen entendimientos y formas de pensar sobre la investigación, su rol, alcance y maneras de hacer. Todas ellas respaldadas por una amplia gama de prácticas e instituciones que trazan el terreno donde es posible hacer y pensar de la forma en la que lo hacemos dentro de un sistema de razón. Por otra parte, nos invita a cuestionar cómo se forma y regula la conducta de investigadores, investigados, lectores y consumidores a través de las prácticas de investigación. La investigación se ha establecido como un sistema que produce conocimiento, al seguir el método científico y al responder a un régimen de poder, de manera tal que se hace plausible sostener su desarrollo. 


\section{INVESTIGACIÓN Y PRODUCCIÓN DE CONOCIMIENTO EN EL ÁREA}

Siempre que hacemos investigación, diseñamos una estrategia para el proceso de construir conocimiento sobre los objetos involucrados en nuestro estudio. Es obvio decir que estas estrategias dependen de los marcos teóricos y metodológicos, lo mismo que de las tradiciones del campo de estudio. En la educación matemática como campo de práctica de investigación que se enfoca principalmente en la triada didáctica [...] El movimiento de investigación ha estado encaminado bacia una exploración en profundidad de pocos factores y actores. El resultado de tal movimiento ha sido la producción de una cantidad considerable de conocimiento sobre cómo funcionan los factores de manera aislada, a expensas de cómo interactúan todos juntos. (Valero, 2012, p. 322)

Parecería que dentro de la educación matemática existe una narrativa dominante con respecto a lo que se considera investigación — de calidad, válida y valiosa - , la cual sustenta lo que se considera como tradiciones del campo, así como estrategias investigativas e intereses a investigar. Esta narrativa ha sido respaldada por diversas métricas de revistas (por ejemplo, el factor de impacto de Web of Science) y, también, por estudios que han concluido criterios para reconocer una investigación de calidad, ya sea éstas empírica o teórica. Un ejemplo de estos criterios se presenta en el trabajo de Schoenfeld (2007), quien afirma que la confiabilidad de una investigación de calidad depende de su (a) poder descriptivo y exploratorio, (b) predicción y falsificación, (c) rigor y especificidad, (d) replicabilidad y (e) triangulación. Esta narrativa reduce las posibilidades de pensar en algo diferente tanto teórica y metodológicamente hablando, así como de considerar otros intereses, preocupaciones, factores y actores que constituyen la red de prácticas sociales (Valero, 2012) en la que se posiciona la educación matemática.

Si bien el giro social (Lerman, 2000) y sociopolítico (Gutiérrez, 2013) en educación matemática han posibilitado nuevas problematizaciones en el área, al colocar en el centro de la educación matemática otro tipo de inquietudes e intereses teóricos y metodológicos (Valero, 2009). En estos giros, las investigaciones se han centrado más en estudiar las condiciones culturales, los arreglos sociales y las decisiones políticas que influyen en la forma en que los estudiantes, profesores, investigadores y responsables políticos se relacionan con la educación matemática, principalmente desde cuestiones de justicia social y equidad (Pais, 2018). Este hecho ha alimentado una tendencia en la investigación a centrarse mayormente en cuestiones internas de la enseñanza y el aprendizaje de las matemáticas (Lerman, 2014), donde “existe la sensación de que tenemos que prescribir o al menos identificar buenas prácticas” (Jablonka et al., 2013, p. 47). En esta línea, parece que el objetivo principal de la investigación es desarrollar, producir y reproducir experiencias exitosas (Gutiérrez, 2013), con el fin de promover soluciones (Pais, 2012) a diversos problemas de la enseñanza y aprendizaje de las matemáticas, y no reflexionar sobre sí misma. Se termina, de cierta forma, reduciendo toda problemática en la búsqueda de mejores formas de enseñar y aprender matemáticas, desplazando del foco de atención y discusión 
aspectos propios de un territorio, como es el caso de una cosmovisión, así como aspectos económicos y políticos, a pesar de ser reconocidos estos a la hora de (re)producir conocimiento. Al respecto, Pais (2012) afirma que "lo que se reconoce como un problema económico y político termina siendo abordado de manera técnica: [con el objetivo de promover] mejores formas de enseñar y aprender matemáticas para todos los estudiantes” (p. 77).

La búsqueda constante por mejorar la enseñanza y el aprendizaje de las matemáticas toma a la investigación como el medio oficial para promover los cambios plausibles, al configurar una narrativa de salvación en torno a ella, la que naturaliza y normaliza el papel de la investigación y lo que se considera como tal. En este contexto, ¿es posible pensar una investigación más allá de la mejorar la enseñanza y el aprendizaje de las matemáticas? Si nos permitimos a responder afirmativamente esta pregunta, ¿aún estaremos dentro de campo de la educación matemática? Además ¿qué espacios —instituciones y medios - tenemos para su divulgación? Si uno posiciona en el centro de red de prácticas sociales (Valero, 2012) al triángulo didáctico, ciertas problematizaciones que no aluden a él de forma directa, como es el caso de estudiar las narrativas de organismos transnacionales o cuestiones de género, por ejemplo, parecieran ubicarse en un territorio periférico a los intereses propios de la educación matemática. Y es aquí donde emerge automáticamente la pregunta: ¿dónde está la matemática (o el contenido matemático) de la investigación? Pero esta pregunta nos muestra lo complejo que puede ser desplazarnos de los terrenos más ortodoxos de la investigación en educación matemática, la centración del triángulo didáctico y, con ello, la mejora de la enseñanza y el aprendizaje de las matemáticas. Por otra parte, al pensar la investigación y la producción de conocimiento pareciera que es imposible hacerlo fuera del positivismo o neopositivismo, así como desde la lógica de mercado y su dinámica. Esto a pesar de que algunos autores han encontrado en las teorías posmodernas un lenguaje para abordar problemas históricamente desconectados de las matemáticas (Pais, 2018). Actualmente, pensar a la educación matemática como potencia y fuente de producción tiende a ser inseparable de su carácter productivo desde una mirada capitalista. En este contexto, pensar la producción de conocimiento más allá del capital implicaría concebirla como no productiva. Por consiguiente, mientras la educación matemática desatiende la importancia de pensar la investigación de otra manera, a partir del problema que implica entenderla como productiva, la investigación tiende a verse a sí misma de una nueva manera —al reconocer e imbricar otros intereses, factores y actores - pero solo para subordinarse a una concepción mercantil de la producción del conocimiento. En otras palabras, la producción de conocimiento ha devenido en una producción para el mercado. Lo que se ve concretado en estándares a lograr al momento de producir conocimiento, como es el caso de la publicación en revistas indexadas con el fin de "validar" la calidad de la investigación y dotándola de un valor. Estándares que se ha vuelto clave para los procesos de toma de decisiones relacionadas con la contratación, retención, promoción y asignación de recursos a los académicos en sus instituciones (Williams \& Leatham, 2017). La configuración de estándares “que se consideran medidas de calidad en la academia, han cambiado la forma en que los académicos deciden 
dónde publicar su trabajo y cómo perciben y leen las revistas” (Andrade-Molina et al., 2020, p. 360), además de establecer métricas de comparación claras y homologas para todos, de forma tal que todos pueden competir con el otro y consigo mismo.

Como se dijo anteriormente, a partir de la relación entre la producción de conocimiento y el desarrollo de la educación matemática se puede, por una parte, reconocer la emergencia de nuevos cuestionamientos, lo que conlleva poner la atención en aspectos externos a la — supuesta - lógica de la investigación o forma de hacer en el área, pero a pesar de ello se continúa pensando de la misma manera. Por otra parte, es posible concebir nuevas formas de hacer y entender la investigación en búsqueda de insubordinarse a la concepción mercantil de producción. Como nos muestra Pais (2018), tomando como base los trabajos desarrollados por de Freitas, la investigación biosocial desarrollada en el campo se

constituye un caso tipico del impulso del capital hacia la acumulación, donde se conciben nuevas tendencias, nuevas metodologias, nuevas pedagogias como una actuación contra la imposibilidad de la educación. [...] el marco más que bumano sobre el que descansa la investigación biosocial de Freitas contradice su propia percepción de cómo la investigación biosocial puede ser rescatada con propósitos inclusivos (p. 376).

Tanto al reconocer la emergencia de nuevos cuestionamientos como al concebir nuevas formas de hacer y entender la investigación en el área, pareciera que la educación matemática tiende a ser indiferente con respecto a ir más allá de lo establecido o considerado como los límites de lo que es posible y no es posible pensar y hacer. La priorización de ciertas corrientes y problemáticas abordadas dentro del área, e incluso de formas de escribir y reportar la investigación, ha establecido una especie de monopolio dentro del campo. Este monopolio conduce a creer que todo lo que no se encuentre enmarcada en estas corrientes, problemas y formas de investigar es por completo ajeno a la agenda que la educación matemática debe hacer frente, por ello poco o nada tiene que aportar.

$\mathrm{Al}$ poner en operación un entendimiento sobre ciencia y producción de conocimiento desde los trabajos de Deleuze y Guatari (1977), lo que sin duda es aplicable a la educación matemática. Es posible desplazarnos a pensar a la ciencia como una actividad de creación —es decir de producción y no de descubrimiento-, así como a la producción más allá de la idea de producir un producto a favor de una caracterización hecha en término de proceso. Con ello, nos veremos forzados a romper con lo que se ha establecido como lo científico - en termino de teorías, entendimientos y formas de entender el mundo-, así como también con la actividad misma de producción científica de conocimiento. Consecuentemente, se hace necesario repensar los conceptos de ciencias, de producción, así como su relación. Ir más allá de las fronteras conocidas de la disciplina se vuelca en un compromiso ético, con respecto a lo que nuestro trabajo como educadores e investigadores tiene para ofrecernos y ofrecerles a las generaciones venideras (Valero, 2012). 
Si consideramos que todo es producción (Deleuze \& Guattari, 1977) y esta producción es una categoría económica, política y social, pero sobre todo ontológica, entendiéndola como una variación constante y abierta - no es una finalidad, ni un fin. El producir y producto no son dos cosas separadas, más bien el producto es un efecto inminente de la producción, siendo la distribución y el consumo las dimensiones internas de la producción.

\section{UNA CUESTIÓN DE ESTÉTICA}

Durante los últimos cincuenta años, lo que se ha publicado bajo la etiqueta de investigación en educación matemática constituye un cuerpo muy amplio, diverso y extenso de contribuciones al campo. [...] Sin embargo, durante las últimas tres décadas he detectado un espectro de variación gradualmente reducido en la naturaleza de las investigaciones publicadas "convencionales"(Niss, 2018, p. 35).

Pareciera que existe una forma correcta de hacer una investigación compartida y aceptada por todos, que es de sentido común, lo que podría justificar el reducido espectro de variación que ha tenido la investigación en las últimas décadas. El no cuestionar esta forma de hacer investigación, y con ello perpetuar el cómo concebimos la investigación — buena y deseada-, se vuelve "perjudicial y potencialmente peligroso para el desarrollo y futuro de nuestro campo" (Niss, 2018, p. 47), dado que no permite pensar otras formas de hacer, otras problemáticas y otras preocupaciones que favorezcan el desarrollo de nuevos entendimientos y conocimientos.

En el acto de investigar se ha articulado una especie de normativas o estándares que definen cómo escribir un artículo o reporte de investigación y qué tópicos o problemáticas deben abordarse y de qué forma hacer este abordaje, teórica y metodológicamente hablando. De esta manera, se gobierna a los educadores matemáticos desde lo que se configura como investigación válida y de calidad —deseada—, así como los posibles campos de acción.

Ahora bien, partiendo de la estética como una forma de estudio de la belleza (aquello que agrada a los sentidos, lo que se desea), así como desde las ideas foucaultianas de la estética de la existencia, cabe cuestionarse: ¿Qué hace que una investigación sea buena y deseada (bella)? ¿Cómo el educador matemático se constituye en una red de poder - en la que circula esta idea de belleza-? Con respecto a la primera pregunta, la educación matemática, como campo de estudio, ha buscado el perfeccionamiento del conocimiento desde lo que se establece como bueno y deseado — sus cualidades de belleza. Desde una discusión estética, el cómo entendemos y pensamos que debe desarrollarse y divulgarse nos lleva hacia lo sensible. A su vez, cómo percibimos la investigación nos lleva hacia las sensaciones. Con respecto a la segunda pregunta, el devenir del educador matemático se da dentro de una red en la que se enreda lo deseado y temido, trazando un sujeto normal —un educador matemático que produce y/o consume conocimiento dentro de ciertos parámetros - mediante criterios de belleza 
establecidos y prácticas que estos hacen posible. En este contexto, el sujeto es conducido a aprender a regular su propia conducta para producir y consumir cierto tipo de investigación: la investigación publicable y deseada.

¿Es posible encontrar trabajos investigativos diferentes dentro de la educación matemática que rompen con la estática establecida-? Sí, pero no es tarea fácil, ya que no se cuenta con suficientes espacios que permitan y validen estudios que se desprendan de los cánones establecidos, es decir, de una estética, con ello lograr trascender a la forma imperante de pensar y concebir una investigación para que sea considerada bella y deseable. Algunos ejemplos de estos trabajos, que sin duda nos posibilita otras formas de conocer cuestiones propias del área, son, por un lado, el artículo de Andrade-Molina (2018), titulado "Mindniac. The reazonable citizen of schooling (Chilean edition)", el cual tiene la peculiaridad de estar escrito como un comic. Por otro lado, tenemos la presentación plenaria de Cabral y Baldino (2019) durante la décima versión del Mathematics Education and Society Conferences - y en su posterior publicación en el acta de la conferencia-, que hace uso de una narrativa en la que diferentes voces dialogan y se cruzan. Para lograr esto, durante la plenaria dos personas de la audiencia personificaron diferentes voces junto con las de los autores.

El deseo, por su parte, "se entiende como una fuerza activa primaria más que como una respuesta reactiva a una necesidad insatisfecha" (Patton, 2000, p. 70). En este entendimiento, el deseo produce la realidad (Deleuze \& Guattari, 1977), al dar forma a las condiciones de posibilidad donde la investigación en educación matemática es plausible, además de trazar lo aceptable y valorado. La investigación deseada es una formación discursiva que cambia de acuerdo con las condiciones temporales y espaciales, aunque últimamente no se han registrados grandes desplazamientos en las formas de hacer y entenderla, como lo reporta Niss (2018). Siguiendo la idea de devenir de Deleuze y Guattari (1994), se puede considerar que la investigación no es lo que es, más bien, es en lo que se convertirá —en su devenir-. Lo medular aquí es que este devenir es parte de un mundo estetizado, en el que lo bello se consume y no se problematiza, sujetando la (re)producción de conocimiento, así como sujetando y subjetivando al educador matemático. En este sentido, la investigación trabajará sobre el investigador tanto como el investigador trabaja en la investigación.

Desde la estética de la existencia, el objeto sobre el que trabaja el investigador es su vida. Por tanto, si el investigador se separara de su investigación, dejaría de ser investigador; el proceso de investigación se vuelve co-extensivo con la vida del investigador. Teniendo esto en mente, responder a la pregunta: ¿Qué es y qué no es una investigación? No es un asunto trivial, nadie podría refutar que las ideas impregnadas en un artículo "ideal” de investigación publicado en alguna revista es — producto de- una investigación y que, a través de esta, se está produciendo o reproduciendo algún conocimiento. Pero qué pasa con todo lo que escribe un investigador, por ejemplo, borradores, ideas sueltas en algún bloc de notas, artículos rechazados, etc. Será que todos estos productos se puede considerar investigación o conocimiento, estamos hablando de la misma persona escribiendo y pensando, en algunos casos, en el 
mismo espacio y con los mismos recursos que produjo aquel artículo. Acaso los escritos del investigador solo se convierten en investigación - y con ello produce o reproduce conocimiento - al momento en que es formalizado por una institución —en este caso las revistas de educación matemática. Es decir, el trabajo del educador matemático deviene investigación solo porque una institución le dio esa categoría, al igual que el mingitorio de Marcel Duchamp —obra llamada La fuente, que se ha convertido en un icono del dadaísmo como la primera obra conceptual— que es considerado una obra de arte.

La estética que da forma a la investigación en educación matemática y su reproducción nos traza un territorio en el cual las condiciones de posibilidades son dibujadas. Sin embargo, debemos intencionar desplazamientos desde nuestra acción creativa y desde la insubordinación para romper con lo establecido al deslocalizar del centro al aula, así como, hacer difusas aquellas fronteras que distingue un adentro de un afuera y su jerarquización.

\section{EL AUTOR EN LA (RE)PRODUCCIÓN DE CONOCIMIENTO}

Es preciso problematizar al educador matemático como autor, ya que nos lleva hacia una discusión que trasciende a la (re)producción o consumo de conocimiento. Lo que un autor puede enunciar está enmarcado en lo que se ha pensado previamente dentro de una red discursiva y racionalidad. "Eso no quiere decir que todos los individuos que existen dentro de una determinada época estén de acuerdo con una visión particular del mundo, sino simplemente que todas las expresiones y textos sancionados se producen dentro de restricciones discursivas similares” (Mills, 2001, p. 75). Desde un posicionamiento foucaultiano, el autor — en nuestro caso, el educador matemático- no es el origen del discurso (Deleuze, 1986), por lo tanto, lo que él o ella pueda o no decir está ligado a un sistema de razón que circunscribe y determina los discursos (Foucault, 1977). En consecuencia, se puede pensar que las ideas y los conceptos — que son parte de la producción de conocimiento— no son la creación de un solo individuo, es decir, del autor de una investigación. En otras palabras, los discursos no se generan a partir de los descubrimientos científicos de los autores, más bien, los textos de investigación revelan la convergencia de una compleja red de prácticas discursivas. En esta línea, "la función de un autor es caracterizar la existencia, circulación y funcionamiento de ciertos discursos dentro de una sociedad” (Foucault, 1977, p. 124). Esto significa que cada vez que los investigadores escriben sobre sus hallazgos, siempre lo hacen dentro de las restricciones de los sistemas y organizaciones institucionales que determinan lo que es posible decir (Foucault, 1972).

Lo que se (re)produce en torno a lo que debe ser una investigación y la producción de conocimiento son manifestaciones concretas de dichas restricciones que determinan formas de pensar, hacer y decir, jugando un papel clave en la configuración de condiciones en las que las racionalidades y los regímenes de poder/conocimiento determinan lo deseado — formas válidas de conocer e investigar basadas en el método científico. Dentro de tales regímenes de conocimiento, se establece "quién tiene y quién no tiene la autoridad intelectual para decidir asuntos, cómo se debe recopilar información sobre 
quién y por quién, y cosas por el estilo” (Bevir, 1999, p. 66). Por su parte, los regímenes de poder dan forma a "lo que cuenta como un enunciado significativo, qué temas se van a investigar, cómo se van a producir los hechos y cosas por el estilo [...] Todos los regímenes de poder están constituidos por formaciones discursivas" (Bevir, 1999, p. 66).

\section{A MODO DE CIERRE}

Continuar perpetuando la supremacía y hegemonía que tienen algunas formas de entender y pensar la investigación en el campo puede tornarse especialmente problemático al ocultar un discurso de neutralidad. La incapacidad de problematizar lo que se entiende por investigación y (re)producción de conocimiento dentro de la educación matemática es reflejo de la crisis de un pensamiento que ya no puede pensar fuera de lo que se asume como permitido.

Al no cuestionar la (re)producción de la investigación en el campo se pierde la posibilidad de alcanzar o de levantar nuevos entendimientos, en donde el objeto teórico no se vuelva la esencia de la educación matemática, más bien lo teórico — su conceptualización y usos- sirva de insumo y bases para romper con el orden del proceso investigativo. Con ello, concebir que la investigación es más que la representación de la realidad, es

un elemento que forma y construye aquella realidad social al ser el conocimiento experto que formula maneras particulares de pensar y de actuar sobre los objetos y sujetos de investigación. [... Así como] genera marcos discursivos y racionalidades sobre lo que se considera como educación matemática en tiempos y lugares determinados. (Valero, 2017, p. 103)

Por otra parte, Pais (2018), desde una posición lacaniano, afirma que una de las características que se puede identificar dentro de la ciencia moderna es su lógica de acumulación, bajo los auspicios de la economía capitalista.

Dentro del capitalismo, cualquier medida tiene que producir plusvalia; de lo contrario, se descarta por obsoleto, contra las reglas del mercado, incluso inmoral o poco ético. Y lo mismo ocurre con la ciencia. Cualquier resultado cientifico que amenace la homogeneidad de la ciencia, su corpus de verdad, resulta en una crisis. La ciencia moderna se construye como un régimen acumulativo de conocimiento, en la medida en que la economia capitalista tiene en su núcleo la acumulación de capital. Cualquier amenaza a este ciclo de acumulación se considera irracional y retrógrada. (Pais, 2018, pp. 380-381)

Las normas que se establecen para delinear lo que se entiende como investigación y producción de conocimiento no tienen autoridad por sí mismas, éstas se configuran como parte de las redes de poder. 
“[El] poder considerado abstractamente, no 've' ni 'habla' [... más bien] se ejerce a partir de innumerables puntos [...]. Pero precisamente porque ni habla ni ve, hace ver y hablar” (Deleuze, 1986, p. 111). En consecuencia, el sujeto que conoce —educador matemático como sujeto que participan libremente en determinada técnica o ejercicio del poder - , los objetos (eventos y problemáticas) a conocer, las formas y modos de conocer son implicaciones - efectos- del poder y sus devenires. La investigación y producción de conocimiento han constituido objetos, conceptos y técnicas, así como al sujeto mismo.

La estética se puede entender como parte del desarrollo de las nociones de libertad en el mundo moderno - una manifestación en si misma-. El sujeto que tiene gusto, capacidad de juzgar por sí mismo, de crear y pensar libremente deviene como sujeto deseado en la sociedad. Sin embargo, se vuelve paradójico este sujeto deseado, al vernos inmersos en un mundo totalmente estetizado - que es parte de la cultura de masa en la que circulamos-, en donde aquello que se piensa, consume y hace, entre otros, están normalizados por un deber ser y hacer que se traza dentro de una amalgama de relaciones de poder, en la cual se entrecruzan intereses personales, sociales y políticos. En este contexto, los trabajos de Andrade-Molina (2018) y Cabral y Baldino (2019) se vuelven relevantes ya que nos permiten aproximarnos a procesos de conocimiento diferentes, nos abren las puertas a nuevas posibilidades, con las cuales considerar la (re)producción de conocimiento como un lugar formativo y reflexivo, y no como una forma para perpetuar una estética.

¿Por qué es útil abrir la discusión desde la estética en el ámbito de la investigación y producción de conocimiento? La respuesta podría radicar en la inutilidad misma de la investigación y de la producción de conocimiento. En un mundo sujetado por el imperativo de la eficacia y productividad, la experiencia estética y el goce parecieran desdibujarse. Sin embargo, el enfrentarse a una investigación que nos permite una relación distinta, en donde el foco se desplaza desde la eficacia y productividad hacia la experiencia, nos posibilitaría experimentar lo estético de una nueva forma y no solo usarlo como objetivo para buscar lo bello. Los planos de la sensación y el pensamiento están conectados, los objetos y la dimensión simbólica se vuelven un lugar para comunicar. Como educadores matemáticos, no solo comunicamos lo que las investigaciones tienen como objetivo, si no aquellas posibilidades que abre y cierran nuestras decisiones, como es el caso de dónde se publica, en qué idioma se hace y con quiénes lo hacemos, entre otros aspectos. Se comunica más que lo declarado por la investigación.

La investigación y la producción de conocimiento ha caído en una relación superficialmente estética. Una investigación toma valor en función de la(s) institución(es) que la valida, como es el caso de la revista en la que se publica. De momento hemos llegado al punto de no importa — tanto- la investigación en sí, más bien acentuar métricas — de calidad—, como es la indexación de una revista. Esta situación nos lleva, como educadores matemáticos, a perder la capacidad de reacción, en el sentido de que pocas investigaciones nos provocan algo, ya que estamos acostumbrados a un tipo de producción y difícilmente nos encontraremos con algo diferente. Si pensamos la investigación desde la estética, ella tiene que ver con las emociones y lo sensible, la investigación debe generar todo tipo de experiencias, nos debe incomodar, agradar, generar 
ambigüedades - placer y molestia, por ejemplo- y principalmente cuestionamientos; no solo debe darnos espacio para lo esperado, lo que aceptamos — en mayor o menor medida - como investigación. De esta forma la investigación debe abrirnos posibilidades de acción y reacción.

Entonces desde una discusión de la belleza - bueno y deseado- en la investigación y producción de conocimiento, nos queda la pregunta: ¿Cómo reivindicar nuestro quehacer autónomo- como sujetos políticos y éticos? Y más aún, ¿cómo podemos usar la belleza como mecanismo subversivo?

\section{ACLARATORIAS}

El autor no tiene conflicto de interés que declarar. El artículo ha sido financiado con recursos propios del autor.

\section{REFERENCIAS}

Andrade-Molina, M. (2017). (D)effecting the child: the scientifization of the self through school mathematics [tesis de doctorado, Aalborg University Press]. https://doi.org/10.5278/vbn.phd.eng.00032

Andrade-Molina, M. (2018). "Mindniac". The reasonable citizen of schooling (Chilean Edition). The Mathematics Entbusiast, 15(1), 36-53.

Andrade-Molina, M., Montecino, A., \& Sánchez, M. (2020). Beyond quality metrics: defying journal rankings as the philosopher's stone of mathematics education research. Educational Studies in Mathematics, 103(3), 359-374. https://doi.org/10.1007/s10649-020-09932-9

Bevir, M. (1999). Foucault and critique: deploying agency against autonomy. Political Theory, 27(1), 65-84. https://doi.org/10.1177/0090591799027001004

Cabral, T., \& Baldino, R. (2019). The social turn and its big enemy: a leap forward. En J. Subramanian (Ed.), Proceedings of The Tenth International Mathematics Education and Society Conference (pp. 32-46). MES10.

Deleuze, G. (1986). Foucault (S. Hand, ed. y trad). The Athlone Press. https://doi.org/10.5040/9781350252004

Deleuze, G., \& Guattari, F. (1977). Anti-oedipus: capitalism and schizophrenia (R. Hurley, M. Seem, $\&$ H. R. Lane, trads.). Viking Press.

Deleuze, G., \& Guattari, F. (1994). What is philosophy? Columbia University Press. 
Deleuze, G. (1992). Conversaciones. 1972-1990. Editorial Pre-textos.

FitzSimons, G. E. (2019). Adults learning mathematics: transcending boundaries and barriers in an uncertain world. Adults Learning Mathematics: An International Journal, 14(1), 41-52.

Foucault, M. (1972). The archaeology of knowledge (A. M. S. Smith, trad.). Pantheon books.

Foucault, M. (1977). What is an author. En D. F. Bouchard (Ed.), Language, Counter-Memory, Practice: Selected Essays and Interviews (pp. 113-138). Cornell University Press.

Foucault, M. (1991). Governmentality. En G. Burchell, C. Gordon, \& P. Miller (Eds.), The Foucault Effect: Studies in Governmentality (pp. 87-104). University of Chicago Press.

Gutiérrez, R. (2013). The sociopolitical turn in mathematics education. Journal for Research in Mathematics Education, 44(1), 37-68. https://doi.org/10.5951/jresematheduc.44.1.0037

Gutstein, E. (2006). Reading and writing the world with mathematics: toward a pedagogy for social justice. Taylor \& Francis.

Gutstein, E. (2012). Mathematics as a weapon in the struggle. En O. Skovsmose, \& B. Greer (Eds.), Opening the Cage: Critique and politics of mathematics education (pp. 23-48). Sense Publishers. https://doi.org/10.1007/978-94-6091-808-7 2

Jablonka, E., Wagner, D., \& Walshaw, M. (2013). Theories for studying social, political and cultural dimensions of mathematics education. En M. A. Clements, A. J. Bishop, C. Keitel, J. Kilpatrick, \& F. K. S. Leung (Eds.), Third International Handbook of Mathematics Education (vol. 27, pp. 41-67). Springer. https://doi.org/10.1007/978-1-4614-4684-2 2

Lee, C. J., Sugimoto, C. R., Zhang, G., \& Cronin, B. (2013). Bias in peer review. Journal of the American Society for Information Science and Technology, 64(1), 2-17. https://doi.org/10.1002/asi.22784

Lerman, S. (2000). The social turn in mathematics education research. En J. Boaler (Ed.), Multiple perspectives on mathematics teaching and learning (pp. 19-44). Ablex.

Lerman, S. (2001). A review of research perspectives on mathematics teacher education. En F. L. Lin, \& T. J. Cooney (Eds.), Making Sense of Mathematics Teacher Education (pp. 33-52). Springer. https://doi.org/10.1007/978-94-010-0828-0 2

Lerman, S. (2014). Mapping the effects of policy on mathematics teacher education. Educational Studies in Mathematics, 87(2), 187-201. https://doi.org/10.1007/s10649-012-9423-9 
Meaney, T. (2013). The privileging of english in mathematics education research, just a necessary evil? En M. Berger, K. Brodie, V. Frith, \& K. le Roux (Eds.), Proceedings of the Seventh International Mathematics Education and Society Conference (pp. 65-84). Hansa Print.

Mills, S. (2001). Discourse. Routledge.

MINEDUC. (2012). Estándares orientadores para carreras de pedagogía en educación media. LOM Ediciones.

MINEDUC. (2021). Programa de Estudio Física $3^{\circ}$ o $4^{\circ}$ medio. Formación diferenciada ciencias, Física. Ministerio de Educación.

Niss, M. (2018). The very multi-faceted nature of mathematics education research. En E. Bergqvist, M. Österholm, C. Granberg, \& L. Sumpter (Eds.), Proceedings of the $42 \mathrm{nd}$ Conference of the International Group for the Psychology of Mathematics Education (vol. 1, pp. 35-50). PME.

Niss, M., Blum, W., \& Galbraith, P. (2007). Introduction. En W. Blum, P. L. Galbraith, H.-W. Henn, \& M. Niss (Eds.), Modelling and Applications in Mathematics Education: The 14th ICMI Study (pp. 3-32). Springer Publishing. https://doi.org/10.1007/978-0-387-29822-1 1

Nivens, R. A., \& Otten, S. (2017). Assessing journal quality in mathematics education. Journal for Research in Mathematics Education, 48(4), 348-368. https://doi.org/10.5951/jresematheduc.48.4.0348

OCDE. (2010). Mathematics teaching and learning strategies in PISA: Programme for International Student Assessment. OECD Publishing.

OCDE. (2014a). Education at a glance 2014: OECD indicators. OECD Publishing.

OCDE. (2014b). PISA 2012 results in focus. What 15-year-olds know and what they can do with what they know. OECD Publishing.

OCDE. (2014c). PISA 2012 results: what students know and can do - Student performance in mathematics, reading and science (Volume I, Revised edition, February 2014). OECD Publishing.

OCDE. (2019a). PISA 2018 Results (Volume I): what students know and can do. OECD Publishing.

OCDE. (2019b). PISA 2018 Results (Volume II): where all students can succeed. OECD Publishing. 
OCDE. (2019c). PISA 2018 Results (Volume III): what school life means for students' lives. OECD Publishing.

Pais, A. (2012). A critical approach to equity. En O. Skovsmose, \& B. Greer (Eds.), Opening the Cage: Critique and politics of mathematics education (vol. 23, pp. 49-91). Sense Publishers.

Pais, A. (2018). Mathematics, capitalism and biosocial research. Educational Studies in Mathematics, 101(3), 373-386. https://doi.org/10.1007/s10649-018-9859-7

Pais, A., \& Valero, P. (2012). Researching research: mathematics education in the Political. Educational Studies in Mathematics, 80(1), 9-24. https://doi.org/10.1007/s10649-012-9399-5

Patton, P. (2000). Deleuze and the Political. Routledge.

Popkewitz, T. S. (2011). The past as the future of the social and education sciences. En D. Tröhler, \& R. Barbu (Eds.), Education Systems in Historical, Cultural, and Sociological Perspectives (pp. 163180). Sense Publishers. https://doi.org/10.1007/978-94-6091-827-8 11

Popkewitz, T. S., Diaz, J., \& Kirchgasler, C. (2017). The reason of schooling and educational research: culture and political sociology. En T. S. Popkewitz, J. Diaz, \& C. Kirchgasler (Eds.), A Political Sociology of Educational Knowledge: Studies of Exclusions and Difference (pp. 3-22). Routledge. https://doi.org/10.4324/9781315528533-1

Schoenfeld, A. H. (2007). Problem solving in the United Status, 1970-2008: research and theory, practice and politics. ZDM: The International Journal on Mathematics Education, 39(5-6), 537551. https://doi.org/10.1007/s11858-007-0038-z

Smid, H. J. (2014). History of mathematics teacher education. En A. Karp, \& G. Schubring (Eds.), Handbook on the History of Mathematics Education (pp. 579-595). Springer. https://doi.org/10.1007/978-1-4614-9155-2 29

Stahnke, R., Schueler, S., \& Roesken-Winter, B. (2016). Teachers' perception, interpretation, and decision-making: a systematic review of empirical mathematics education research. ZDM: The International Journal on Mathematics Education, 48(1), 1-27. https://doi.org/10.1007/s11858$\underline{016-0775-y}$

Toerner, G., \& Arzarello, F. (2012). Grading mathematics education research journals. Newsletter of the European Mathematical Society, 86, 52-54.

Valero, P. (2009). What has power got to do with mathematics education? En P. Ernest, B. Greer, \& B. Sriraman (Eds.), Critical Issues in Mathematics Education (pp. 237-254). IAP. 
INVESTIGACIÓN Y PRODUCCIÓN DE CONOCIMIENTO EN EDUCACIÓN MATEMÁTICA...

Valero, P. (2012). La educación matemática como una red de prácticas sociales. En P. Valero, \& O. Skovsmose (Eds.), Educación matemática critica. Una visión sociopolitica del aprendizaje y la enseñanza de las matemáticas (pp. 299-326). Una empresa docente.

Valero, P. (2017). El deseo de acceso y equidad en la educación matemática. Revista Colombiana de Educación, 73, 99-128. https://doi.org/10.17227/01203916.73rce97.126

Williams, S. R., \& Leatham, K. R. (2017). Journal quality in mathematics education. Journal for Research in Mathematics Education, 48(4), 369-396.

https://doi.org/10.5951/jresematheduc.48.4.0369

\section{Cómo citar este artículo:}

Montecino, A. (2021). Investigación y producción de conocimiento en educación matemática: una cuestión de mercado, poder y estética. Revista Venezolana de Investigación en Educación Matemática (REVIEM), 1(2), e202109. https://doi.org/10.54541/reviem.v1i2.20 
Alex Montecino

Copyright $@$ 2021. Alex Montecino. Esta obra está protegida por una licencia Creative Commons 4.0. International (CC BY 4.0).

Usted es libre para Compartir — copiar y redistribuir el material en cualquier medio o formato - y Adaptar el documento - remezclar, transformar y crear a partir del material — para cualquier propósito, incluso para fines comerciales, siempre que cumpla la condición de:

Atribución: Usted debe dar crédito a la obra original de manera adecuada, proporcionar un enlace a la licencia, e indicar si se han realizado cambios. Puede hacerlo en cualquier forma razonable, pero no de forma tal que sugiera que tiene el apoyo del licenciante o lo recibe por el uso que hace de la obra.

$\underline{\text { Resumen de licencia - Texto completo de la licencia }}$ 